\title{
Über Strukturisomerie: Darstellung von asymmetrischen Seleniten.
}

\author{
Von \\ L. Marino. ${ }^{1}$
}

Die Theorie von Werner hat den Begriff der Isomerie in die Betrachtung anorganischer Verbindungen eingeführt; sie gestattet die Existenz einer beträchtlichen Anzahl von Isomeren vorauszusehen und zu klassifizieren, die gröfstenteils den Typus der stark komplexen Verbindungen angehören. Unter den mannigfachen Isomeriearten - Polymerie, Stereoisomerie, Salzisomerie, Koordinationsisomerie, Hydratationsisomerie - ist jedoch die Strukturisomerie, die gerade die einfacheren, zum Studium der Molekularumwandlungen sẹh geeigneten Fälle umfalst, bisher weniger berücksichtigt worden.

Meines Erachtens liegt der Grund für die mangelhafte Entwickelung gerade dieses Gebietes in der irrigen Auffassung, dafs bei der Bildung anorganischer Verbindungen das Reaktionsprodukt strukturell unabhängig sein soll von der Natur seiner Komponenten. Bariumsulfat soll zum Beispiel dieselbe Zusammensetzung und Struktur besitzen, gleichgültig, ob es durch Einwirkung von Schwefelsäure auf Bariumoxyd oder von Schwefeldioxyd auf Bariumsuperoxyd entsteht; oder mit vaN'т HoFfs Worten: „Es fehlt bei anorganischen Verbindungen die eigentümliche Starrheit, welche dem Produkt die Merkmale seines Ursprunges aufzwingt, fast ganz."2 Die folgenden Ausführungen lassen aber erkennen, dafs auch bei der Bildung gewisser anorganischer Verbindungen eine gewisse Abhängigkeit besteht und dafs es wahrscheinlich nur an der Unvollkommenheit unserer Untersuchungsmethoden und an der Schwierig-

${ }^{1}$ Aus dem Italienischen übersetzt von R. J. Merer.

${ }^{2}$ Van't Hoff, Vorlesungen über theoret. u. physikal. Chemie II, S. 79, Braunschweig 1903. 
keit die Bildungsbedingungen ausfindig zu machen liegt, wenn der Nachweis der Verschiedenheit chemischer Individuen häufig unmöglich erscheint.

Behufs experimenteller Prüfung dieser Erwägungen habe ich eine Untersuchung unternommen, deren erste Resultate in vorliegender Abhandlung gegeben werden. Sie sind geeignet die oben entwickelten Ideen $\mathrm{zu}$ bestätigen und lehren auch einen bemerkenswerten Fall von Isomerie kennen.

Aus den beim Studium der Reaktion: ${ }^{1}$

$$
\mathrm{PbO}_{2}+\mathrm{SO}_{2}=\mathrm{PbSO}_{3}+\mathrm{O}
$$

erhaltenen Resultaten im Zusammenhange mit den damals entwickelten Betrachtungen schlofs ich, dafs das so erhaltene Bleisulfit asymmetrische Struktur haben müsse. Es ist nun bekannt, dafs gewisse einfache Säuren, zum Beispiel salpetrige, schweflige und vielleicht auch phosphorige Säure ${ }^{2}$ tautomer auftreten; sie können daher isomere Derivate geben, die sich beispielsweise von folgenden Strukturformeln ableiten lassen:

$$
\mathrm{O}=\mathrm{N} . \mathrm{OR} \text { und } \stackrel{\mathrm{O}}{\mathrm{O}} \gg \mathrm{NR} ; \quad \mathrm{O}=\mathrm{S}<<_{\mathrm{OR}}^{\mathrm{OR}} \text { und } \stackrel{\mathrm{O}}{\mathrm{O}} \gg \mathrm{S}<_{\mathrm{R}}^{\mathrm{OR}}
$$

Nun kennt man zwar die entsprechenden Alkylderivate dieser Substanzen, dagegen lassen sich von ihnen keine isomeren Metallsalze darstellen, vielleicht aus dem Grunde weil, wie $\mathrm{H}_{\text {Antzsch }}{ }^{3}$ annimmt, die meist stark positiven Metalle das Bestreben haben die tautomere Säure energisch im Sinne der negativen Atomgruppierung umzuwandeln. Ist dies richtig, so mülste man, ebenso wie ich es für die schweflige Säure gezeigt habe, auch von der selenigen Säure ausgehend, zu einem asymmetrischen Selenit kommen. Da nun die nach den gewöhnlichen Darstellungsmethoden gewonnenen Metallselenite symmetrische Struktur besitzen, da sie alle zu demselben Ester führen, so müssen sich in diesem Falle Isomere darstellen. lassen, die der tautomeren Formel II entsprechen:

I. $O=\operatorname{Se}<\begin{aligned} & O R \\ & O R\end{aligned}$ II. $\quad \mathrm{O}_{\mathrm{O}} \gg \mathrm{Se}<\frac{\mathrm{OR}}{\mathrm{R}}$.

1 Marivo, Z. anorg. Chem. 56 (1907), 234.

${ }^{2}$ Hantzsch, Z. f. Elektrochem. 8, 484.

${ }^{3}$ Hantzsch, Ber. deutsch. chem. Ges. 35 (1902), 2717. 


\section{Einwirkung von seleniger Säure auf Bleidioxyd.}

Lälst man selenige Säure auf Bleidioxyd einwirken, so ist das Ergebnis ein verschiedenes, je nach den Versuchsbedingungen. Wendet man verdünnte Säure an, so findet auch bei langem Kochen keine Reduktion des Dioxyds statt. Bei mittlerer Konzentration vollzieht sich zwar die Reduktion schnell, doch erhält man das gewöhnliche, symmetrische Selenit neben kleinen Mengen von Seleniat. Bei starker Säurekonzentration bildet sich ein Gemisch des gewöhnlichen Selenits mit einem gelben Salz, das nach der weiter unten beschriebenen Methode rein dargestellt werden kann. Die Reduktion wurde analog wie früher im Falle der schwefligen Säure bei Gegenwart von Natriumbiselenit vorgenommen, um die Selensäure in dem Malse, als sie sich bildet, zu binden. Man wendet feuchtes Dioxyd an, weil dasselbe trocken nur äufserst langsam bei stärkster Konzentration der Säure reagiert. Um mit einiger Sicherheit die angewandte Menge $\mathrm{PbO}_{2}$ bestimmen und danach die $\mathrm{SeO}_{2}$ berechnen zu können, liefs man das Dioxyd auf Filtrierpapier liegen, bis es eben noch feucht war. In einer kleinen Quantität wurde der Gehalt an $\mathrm{PbO}_{2}$ in der üblichen Weise bestimmt, worauf sofort die für die verschiedenen Versuche anzuwendenden Mengen abgewogen wurden. Das Selenigsäureanhydrid wurde zweimal sublimiert, nachdem vorher die vorhandenen Spuren von Schwefelsäure mit Baryt entfernt worden waren. ${ }^{1}$

Die Reaktion wurde folgendermafsen ausgeführt: In einem Gläschen wurde die selenige Säure in wenigen Kubikzentimetern Wasser gelöst und hierzu in kleinen Portionen soviel krystallisierte Soda zugefügt, dafs der gröfste Teil der Säure als Biselenit gebunden wurde. Nach Zusatz des feuchten Dioxyds wurde unter fortwährendem Rühren zum Sieden erhitzt, bis das braune Oxyd verschwunden war. Hierzu genügen im allgemeinen 10-15 Minuten. Das erhaltene Produkt (A) wurde nach dem Erkalten abfiltriert und im Filtrat (B) die Selensäure bestimmt. $\mathrm{Zu}$ diesem $\mathrm{Z}$ wecke wurde die Lösung mit Essigsäure angesäuert, worauf die selenige Säure durch Sättigen mit $\mathrm{H}_{2} \mathrm{~S}$ entfernt wurde. Nach dem Filtrieren und Auswaschen des Niederschlages wurde zur Verjagung der Essigsäure und des Schwefelwasserstoffs gekocht und dann mit Salzsäure und Jodkalium nach den Angaben von Gooch und PeIrCE ${ }^{2}$ behandelt.

1 Thomson, Ber. deutsch. chem. Ges. 2, 598.

2 Gooch und Peiree, Z. anorg. Chem. 11 (1896), 249. 
Aus der gewogenen Menge Selen berechnete man die vorhandene Selensäure.

In dem Produkt (A) wurde ebenfalls die in der Form von Bleiseleniat vorliegende Selensäure bestimmt. Man kochte zu diesem Zwecke mit einer Lösung von Kaliumcarbonat und behandelte nach dem Erkalten die mit Wasser verdünnte und filtrierte Lösung wie oben.

Tabelle.

$\mathrm{PbO}_{2}: 0.92 \mathrm{~g}: \mathrm{SiO}_{2}: 1.6 \mathrm{~g}$. $-\mathrm{Nr} \cdot 1$ u. 2 bei Gegenwart von $1.3 \mathrm{~g} \mathrm{Na}_{2} \mathrm{CO}_{3}$. $10 \mathrm{H}_{2} \mathrm{O}$. Nr. 3 u. 4 ohne Alkalizusatz. - Nr. 5 bei Gegenwart von $0.52 \mathrm{~g}$ Ammoninmearbonat.

\begin{tabular}{|c|c|c|c|c|c|c|c|}
\hline & 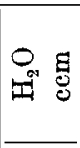 & 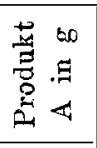 & 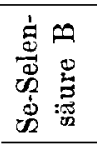 & 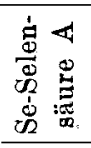 & 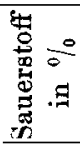 & $\begin{array}{ll}0 & 0 \\
0 & \\
0 & 0 \\
0 & 0 \\
8 & 0 \\
0 & 0\end{array}$ & Bemerkungen \\
\hline 1 & 4 & 1.292 & 0.1895 & 0.0203 & 4.6 & 6.69 & $\begin{array}{l}\text { Direkt erhitzt. - Produkt gelblich } \\
\text { Auf Wasserbad erhitzt. - Pro- }\end{array}$ \\
\hline 2 & 6 & 1.2432 & 0.1864 & Spur. & 4.1 & 6.69 & dukt gelblich \\
\hline 3 & 8 & 1.2260 & 0.1729 & $n$ & 3.80 & 6.69 & $\begin{array}{l}\text { Direkt erhitzt. - Produkt gelblich } \\
\text { Direkt erhifzt. - Produkt weils }\end{array}$ \\
\hline 4 & 16 & 1.2361 & 0.0545 & 0.1789 & 5.13 & 6.69 & $\begin{array}{l}\text { mit gelblichen Punkten } \\
\text { Direkt erhitzt. - Produkt völlig }\end{array}$ \\
\hline 5 & 15 & 1.228 & 0.1698 & 0.0709 & 529 & 6.69 & weifs \\
\hline
\end{tabular}

Die Tabelle zeigt, dals die frei werdende Menge Sauerstoff geringer ist als die Rechnung für den Zerfall von $\mathrm{PbO}_{2}$ in $\mathrm{PbO}+\mathrm{O}$ ergibt. Es zeigt sich ferner, dafs sie sich um so mehr dem berechneten Werte nähert, je weniger das Reaktionsprodukt gelb gefärbt ist. Es ergibt sich aus dem Folgenden und aus der Tatsache, dals man in einigen Fällen die Entwickelung geringer Spuren von Sauerstoff beobachten konnte, wie sich diese kleine Differenz erklären lälst. Durch qualitative Analyse lassen sich im Produkte A drei verschiedene Verbindungen nachweisen, nämlich das gewöhnliche Bleiselenit $\mathrm{PbSeO}_{3}$, Bleiseleniat $\mathrm{PbSeO}_{4}$ und ein gelbes Salz $\mathrm{Pb}_{2} \mathrm{Se}_{2} \mathrm{O}_{7}$, welches nach folgender Methode isoliert und gereinigt wurde:

In einem Becherglas von ungeführ $100 \mathrm{ccm}$ fügte man $\mathrm{zu}$ einer Lösung von $25 \mathrm{~g} \mathrm{SeO}_{2}$ in $25 \mathrm{ccm} \mathrm{H}_{2} \mathrm{O}$ allmählich $20 \mathrm{~g} \mathrm{Na}_{2} \mathrm{CO}_{3}$. $10 \mathrm{H}_{2} \mathrm{O}$ und dann $30 \mathrm{~g}$ feuchtes $\mathrm{PbO}_{2}$ zu $(2 \mathrm{~g}=0.94 \mathrm{~g} \mathrm{PbO})$; man erwärmte vorsichtig mit direkter Flamme bis zum Sieden, ${ }^{1}$ indem

${ }^{1} \mathrm{Da}$ ich über Verletzungen bei Einwirkung konzentrierter Lösungen von $\mathrm{H}_{2} \mathrm{SeO}_{3}$ auf die Haut keinerlei Angaben finde, so möchte ich anführen, dafs 
man das Oxyd mit einem Glasstabe zerdrückte und zerteilte, bis die Masse gleichmälsig schön gelb war. Nach dem Absetzen wurde wiederholt dekantiert, bis die Hauptmenge der selenigen Säure entfernt war. Das gelbe Produkt wurde dann in der Kälte ungefähr 1 Stunde lang mit $250 \mathrm{ccm}$ verdünnter $\mathrm{HNO}_{3}(7-8 \%)$ unter häufigem Rühren digeriert, worauf man noch zweimal mit $100 \mathrm{ccm}$ $\mathrm{HNO}_{3}$ dekantierte und die Salpetersäure mit Wasser auswusch. Auf diese Weise wird sowohl das gewöhnliche Selenit als auch eventuell vorhandenes Seleniat aus dem gelben Produkt entfernt. Schliefslich wird letzteres abgesaugt und bei $100^{\circ}$ getrocknet.

Zur Bestimmung des Selens löste ich ungeführ ein Gramm des Salzes in $\mathrm{HCl}$, verdünnte mit $400 \mathrm{ccm} \mathrm{H}_{2} \mathrm{O}$ und kochte nach $\mathrm{Zu}$ fügung eines Überschusses von Jodkalium. Nach Fortkochen des in Freiheit gesetzten Jods wurde die Flüssigkeit siedend filtriert und das abgeschiedene Selen nach Auswaschen mit siedendem Wasser auf gewogenem Filter bei $100^{\circ}$ getrocknet und gewogen. Im Filtrat wurde das Blei nach dem Eindampfen zur Trockene bestimmt, indem das $\mathrm{J}_{\mathrm{od}}$ mit $\mathrm{HNO}_{3}(1: 1)$ und die Salpetersäure mit $\mathrm{HCl}$ verjagt wurde. Der trockene Rückstand wurde mit soviel Wasser aufgenommen, dals das Bleichlorid in Lösung blieb, worauf man mit einigen Tropfen $\mathrm{HCl}$ ansäuerte und das Blei bei Gegenwart von Natriumacetat als Chromat ausfällte und letzteres auf getrocknetem Filter wog. In einigen Fällen wurde das Blei zur Kontrolle auch als Sulfat bestimmt. Man erhielt so folgende Resultate:

I. $0.7334 \mathrm{~g}$ Substanz ergaben Gramm Se: 0.1710 und Gramm $\mathrm{PbCrO}_{4}: 0.6875$.

II. 0.9630 g Substanz ergaben Gramm Se: 0.2231 und Gramm $\mathrm{PbCrO}_{4}: 0.9070$ (neue Darstellung).

III. 0.8800 g Substanz ergaben Gramm Se: 0.2045 und Gramm $\mathrm{PbCrO}_{4}: 0.8290$ (neue Darstellung).

IV. $0.9472 \mathrm{~g}$ Subst. ergaben Gramm Se: 0.2180

$$
\left.\begin{array}{l}
0.9472 \mathrm{~g} \text { Subst. ergaben Gramm Se: } 0.2180 \\
1.1276 \mathrm{~g}, \quad, \mathrm{PbSO}_{4}: 0.9962
\end{array}\right\} \text { Darst. III. }
$$

ich nach einem versehentlichen einige Minuten dauernden Eintanchen der Spitze des kleinen Fingers in eine Lösung von $\mathrm{H}_{2} \mathrm{SeO}_{3}(30 \%)$, die $8 \% \mathrm{H}_{2} \mathrm{SeO}_{4}$ enthielt, allgemeine Vergiftungserscheinungen an mir beobachtete, die mit Schwindelanfällen und sehr heftigen lokalen Schmerzen anftraten. Die Gefühlsempfindlichkeit stellte sich in dem verbranten Finger erst langsam nach ungefähr 2 Monaten wieder her. Lokal schied sich rotes Selen ab. 
Berechnet für

$$
\begin{aligned}
& \mathrm{Pb}_{2} \mathrm{Se}_{2} \mathrm{O}_{7} \text { : } \\
& \mathrm{Pb}_{2}=413.8 \quad 60.43 \\
& \mathrm{Se}_{2}=158.2 \quad 23.10 \\
& \mathrm{O}_{7}=112.0 \quad 16.47 \\
& 684.0 \quad 100.00
\end{aligned}
$$

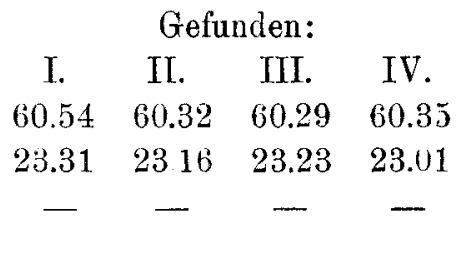

Diese Verbindung ist ein sehr feines krystallinisches Pulver von schwefelgelber Farbe, praktisch unlöslich in Wasser, ver- . dünnter Salpetersäure und Schwefelsäure. In Chlorwasserstoffsäure mittlerer Konzentration löst es sich schnell unter Chlorentwickelung; konzentrierte Salpetersäure verwandelt es bei fortgesetztem Kochen in gewöhnliches Selenit, welches sich beim Zufügen von Wasser klar löst. Aus einer sauren Lösung von Jodkalium wird - auch beim Ansäuern mit ganz verdünnter Essigsäure - sofort Jod frei gemacht. Beim Kochen mit einer Lösung von Kaliumcarbonat entsteht Bleidioxyd. Alkalilauge löst vollständig auf. $\mathrm{H}_{2} \mathrm{~S}$ fällt Bleisulfid, wobei sich gleichzeitig das Selen als Selensulfid ab. scheidet, während sich in der Flüssigkeit keine Spuren von Selensäure finden. Gegen eine Lösung von Oxalsäure verhält sich die Verbindung als kräftiges Oxydationsmittel, indem sie sich in der Siedehitze unter Kohlensäureentbindung löst. Sie ist frei von Krystallwasser und verliert bei mehrstündigem Erhitzen auf 200 bis $250^{\circ} \mathrm{kaum}$ geringe Spuren von seleniger Säure. Diese Reaktionen zeigen, dafs es sich hier um eine einheitliche Verbindung und nicht um ein Gemisch von Bleiselenit und Seleniat handelt. Dafs letzteres nicht zugegen ist, wird auch durch folgende Versuche bestätigt:

Man löste $1.00 \mathrm{~g} \mathrm{PbSeO}_{4}$ in wenigen Minuten in $40 \mathrm{ccm} \mathrm{Am-}$ moniak $(2.7 \%$ ) unter Zusatz von $6 \mathrm{~g}$ Ammoniumtartrat, während bei gleicher Behandlung von $1.00 \mathrm{~g} \mathrm{~Pb}_{2} \mathrm{Se}_{3} \mathrm{O}_{7}$ nur geringe Spuren in Lösung gingen, wahrscheinlich infolge beginnender Zersetzung des Salzes. Das ungelöste Produkt hatte nach dem Auswaschen und Trocknen dieselbe Zusammensetzung wie das Ausgangsmaterial.

\section{Einwirkung von Kaliumcarbonat auf $\mathrm{Pb}_{2} \mathrm{Se}_{2} \mathrm{O}_{7}$.}

Zur quantitativen Bestimmung des bei der Einwirkung von Alkalicarbonaten entstehenden Dioxyds wurde ungefähr $1 \mathrm{~g}$ des Salzes 4-5 Stunden lang mit einer Lösung von $2 \mathrm{~g} \mathrm{~K}_{2} \mathrm{CO}_{3}$ in $50 \mathrm{ccm}$ Wasser digeriert. Das abgeschiedene Dioxyd wurde ausgewaschen und das aus einer Jodkaliumlösung abgeschiedene Jod 
in bekannter Weise mit Natriumthiosulfat titriert. Das aus $1.0583 \mathrm{~g}$ $\mathrm{Pb}_{2} \mathrm{Se}_{2} \mathrm{O}_{7}$ abgeschiedene $\mathrm{PbO}_{2}$ verbrauchte $28.9 \mathrm{ccm} \quad 1 / 10$-norm. Thiosulfat.

$$
\begin{array}{cc}
\text { Gefunden } \mathrm{PbO}_{2}: & \text { Berechnet: } \\
32.8 \% & 36.25
\end{array}
$$

Das heifst: Jede Molekel $\mathrm{Pb}_{2} \mathrm{Se}_{2} \mathrm{O}_{7}$ ergibt bei der Spaltung je eine Molekel $\mathrm{PbO}_{2}$ und $\mathrm{PbO}$. - Auch die Einwirkung von Natriumhydroxyd bestätigt dieses Resultat:

$\mathrm{Pb}_{2} \mathrm{Se}_{2} \mathrm{O}_{7}$ löst sich vollständig in einer $15 \%$ igen Lösung von $\mathrm{NaOH}$. Die klare Lösung wird durch verdünnte Salpetersäure unter Abscheidung von Bleisesquioxyd ${ }^{1}$ gefällt, so dals also die Spaltung nach dem Schema

$$
\mathrm{Pb}_{2} \mathrm{Se}_{2} \mathrm{O}_{7} \longrightarrow \mathrm{Pb}_{2} \mathrm{O}_{3}+2 \mathrm{SeO}_{2} \text { verläuft. }
$$

\section{Verwandlung von $\mathrm{Pb}_{2} \mathrm{Se}_{2} \mathrm{O}_{7}$ in sein Isomeres.}

Die Verbindung $\mathrm{Pb}_{2} \mathrm{Se}_{2} \mathrm{O}_{7}$ bleibt beim Erhitzen bis $140^{\circ}$ unverändert, nur die Färbung wird etwas intensiver. Von $180^{\circ}$ an verblafst die gelbe Färbung langsam und bei ungefähr sechsstündigem Erhitzen auf $200^{\circ}$ entsteht eine weifse Verbindung von gleicher prozentiscber Zusammensetzung:

Angew. Subst. : $0.8330 \mathrm{~g}$ ergab $0.1908 \mathrm{~g}$ Se und $0.7890 \mathrm{~g} \mathrm{PbCrO}_{4}$.

$$
\begin{array}{cc}
\text { Berechnet für } \mathrm{Pb}_{2} \mathrm{Se}_{2} \mathrm{O}_{7}: & \text { Gefunden: } \\
60.43 \% & 60.67 \% \\
23.10 & 22.91
\end{array}
$$

Das so erhaltene weilse Salz unterscheidet sich, abgesehen von der Farbe, auch durch folgende Reaktionen von seinen Isomeren: Es löst sich in verdünnter Salpetersäure in der Kälte. Beim Kochen mit Alkalicarbonaten entsteht nicht Bleidioxyd. Filtriert man die erhaltene Lösung und leitet man in das mit Essigsäure angesänerte Filtrat Schwefelwasserstoff, so fällt nur ein Teil des Selens aus, während der Rest in der Lösung als Selensäure verbleibt.

$0.9804 \mathrm{~g} \mathrm{~Pb}_{2} \mathrm{Se}_{2} \mathrm{O}_{7}$ - völlig weifses Salz -- ergaben Selen (entsprechend der vorhandenen Selensäure) $0.1078 \mathrm{~g}$, entsprechend $48.63 \% \mathrm{PbSeO}_{4}$. Berechnet $51.17 \%$.

1 Über die Zusammensetzung und Struktur dieses Sesquioxyds werde ich in einer folgenden Abhandlung berichten. 
Es ist danach klar, dals in diesem Falle jede Molekel $\mathrm{Pb}_{2} \mathrm{Se}_{2} \mathrm{O}_{7}$ 1 Molekel Bleiselenit und 1 Molekel Bleiseleniat liefern wird. - Mit Oxalsäure entwickelt das weilse Salz keine Kohlensäure; es hat also die oxydierenden Eigenschaften des gelben Salzes verloren. Dals es sich hier nicht um ein durch Zersetzung entstandenes Gemisch aus Selenit und Seleniat handelt, beweist der folgende Versuch:

$0.9863 \mathrm{~g}$ des weifsen, auf $200^{\circ}$ erhitzten, Salzes wurden 72 Stunden mit $6 \mathrm{~g}$ Ammoniumtartrat und $40 \mathrm{ccm}$ Ammoniak $(2.7 \%$ ) digeriert. Der Rückstand wurde abfiltriert, mit Ammoniumtartratlösung gewaschen und in $\mathrm{HCl}$ gelöst und die Lösung mit einem Überschusse von Jodkalium gekocht. Man erhielt so $0.170 \mathrm{~g} \mathrm{Se}=$ $17.23 \%$; berechnet $23.10 \%$.

Lälst man die Verbindung 4-5 Stunden in Berührung mit ammoniakalischem Ammoniumtartrat und verfährt dann in derselben Weise, so erhält man $22.2 \%$ Se; berechnet 23.10. - Unter gleichen Bedingungen gibt ein äquimolekulares Gemenge von Selenit und Seleniat sofort das ganze vorhandene Seleniat ab. Die Differenz wird man daher eher dem Beginne einer Zersetzung als einer partiellen Löslichkeit zuschreiben müssen, die durch die Alkalität der Flüssigkeit hervorgerufen wird. Tatsächlich findet man in der Lösung mehr selenige Säure, als der geringen Löslichkeit des gewöhnlichen Selenits entspricht.

\section{Einwirkung von Kaliumpermanganat auf die beiden Isomeren.}

Um die konstitutionellen Schlüsse, die man aus den beschriebenen Reaktionen ziehen kann, zu bestätigen, wurde die Sauerstoffmenge bestimmt, die das Salz bei der Oxydation aufnimmt. Hierzu eignet sich sehr gut die Oxydation mit Kaliumpermanganat. Bei Anwendung von $0.8-0.95 \mathrm{~g}$ Substanz wurden konstante Werte unter folgenden Bedingungen erhalten: $0.825 \mathrm{~g}$ des weifsen Salzes in $150 \mathrm{ccm}$ Wasser und $9 \mathrm{ccm}$ konzentrierter Schwefelsäure suspendiert, wurden ungefähr $1 / 2$ Stunde bei $40-50^{\circ}$ unter häufigem Umrühren mit $50 \mathrm{ccm} 1 / 10^{-n o r m}$. Kaliumpermanganat digeriert. Man gab dann $50 \mathrm{ccm}^{1} / 10^{-n o r m}$. Oxalsäure zu und titrierte im Filtrat den Überschufs derselben mit Permanganat zurück. Man verbrauchte so $26 \mathrm{ccm} \mathrm{KMnO}_{4}$, woraus sich $2.52 \% \mathrm{O}$ ergaben. (Berechnet $2.34 \%$.)

Das gelbe Salz wird dagegen unter diesen Bedingungen nicht angegriffen; wenn man aber längere Zeit mit $\mathrm{KMnO}_{4}$ und $\mathrm{H}_{2} \mathrm{SO}_{4}$ 
bei $80^{\circ}$ erhitzt, so wird $\mathrm{H}_{2} \mathrm{SeO}_{3}$ in Freiheit gesetzt, die dann zur Oxydation 2 Atome Sauerstoff verbraucht.

$$
\text { Gefunden } \mathrm{O}_{2}=4.20 \% \text {. Berechnet }=4.67 \% \text {. }
$$

Die Differenz dürfte durch kleine Mengen unzersetzter Substanz verursacht sein.

\section{Über die Konstitution der beiden Isomeren.}

Die Gesamtheit aller dieser qualitativen und quantitativen Reaktionen macht folgende Konstitutionsformeln hinreichend wahrscheinlich:

Gelbes Salz:

$$
\mathrm{Pb}-\mathrm{O}-\mathrm{SeO}_{2}
$$

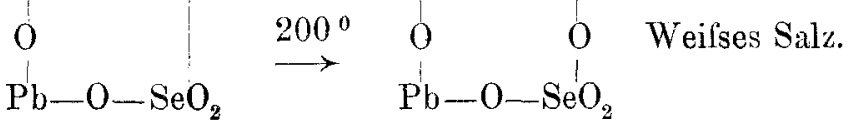

Dieselben stehen gut im Einklang mit der Bildung der verschiedenen Reaktionsprodukte, die bei Einwirkung ron Alkali, Alkalicarbonat, Schwefelwasserstoff und Kaliumpermanganat beobachtet wurden. Sie bringen die Ursache für die Isomerie der beiden Salze und den Zusammenhang zwischen ihrer Bildung und der asymmetrischen Struktur der selenigen Säure deutlich zum Ausdruck. Die Reaktionen des gelben Salzes mit Alkalilauge sowie des weifsen mit Kaliumpermanganat verdienen ein besonderes Interesse, weil man durch Vermittelung der ersteren die Gegenwart des Atomkomplexes $\mathrm{Pb}_{2} \mathrm{O}_{3}$ nachweisen kann, wodurch - da nur selenige Säure zugegen ist - im Zusammenhange mit der Gesamtheit der anderen Umsetzungen, auch die Gruppierung $\mathrm{SeO}_{2}-\mathrm{SeO}_{2}-$ hinreichend wahrscheinlich wird, während die zweite die intramolekulare Wanderung eines Atoms Sanerstoff und die relative Wertigkeitsabnahme des Selens bei der Umwandlung des gelben in das weil'se Salz deutlich macht. Berücksichtigt man ferner, dals sich $\mathrm{Pb}_{2} \mathrm{O}_{3}$ ebenso wie selenige Säure in $\mathrm{HCl}$ löst, ohne dals Cblor frei wird, ${ }^{1}$ und dafs das gelbe Produkt kein Seleniat enthält, so ist es leicht einzusehen, dafs man die beobachteten oxydierenden Eigenschaften ausschliefslich der Gegenwart einer asymmetrischen selenigen Säure zuschreiben mufs. Man muls also die beiden untersuchten Salze als ein erstes Beispiel wahrer an-

\footnotetext{
1 Semel, Journ. pralkt. Chem. [2] 20, 200; siehe auch Dammer II, 2. S. 577.
} 
organischer Strukturisomerie betrachten. Dasselbe verdient um so mehr Interesse, als es sich um Derivate von zwei isomeren Formen derselben Säure handelt. Fälle dieser Art waren bisher in der anorganischen Chemie nicht bekannt, wenigstens, wenn man absieht von den Isomerien: ${ }^{1}$

$$
\begin{gathered}
\mathrm{H}_{\mathbf{2}} \mathrm{N}_{\mathrm{NO}} \\
\text { Nitramid und } \underset{\mathrm{HO}}{\mathrm{H}}>\mathrm{N} . \mathrm{NO} \text {, identisch mit }(\mathrm{HO}) \mathrm{N}=\mathrm{N}(\mathrm{OH}),
\end{gathered}
$$

Verbindungen die $\mathrm{H}_{\mathrm{ANTZSCH}}{ }^{2}$ als stereoisomer auffafst:

$$
\begin{array}{r}
\mathrm{HO}-\mathrm{N} \\
\mathrm{N}-\mathrm{OH}
\end{array} \text { und } \begin{aligned}
& \mathrm{N}-\mathrm{OH} \\
& \mathrm{N}-\mathrm{OH}
\end{aligned} \text {. }
$$

Diese Hypothese von Haxtzsch ist - worauf schon ANGELI ${ }^{3}$ hingewiesen hat - deshalb wenig wahrscheinlich, weil das Nitramid mit Diazomethan als Hauptprodukt das Dimethylnitramid $\left(\mathrm{CH}_{3}\right)_{2} \mathrm{~N} \cdot \mathrm{NO}_{2}{ }^{4}$ gibt, und da es andererseits ein Derivat der Salpetersäure ist, so müssen die beiden Sauerstoffatome an dasselbe Stickstoffatom gebunden sein.

Der von mir studierte Fall regt noch zu einer anderen interessanten Betrachtung an: Läfst man Bleidioxyd auf eine nicht sehr konzentrierte Lösung von $\mathrm{H}_{2} \mathrm{SeO}_{3}$ einwirken, so entsteht als Hauptprodukt das gewöhnliche Bleiselenit, zusammen mit Seleniat und daneben eine geringe Menge $\mathrm{Pb}_{2} \mathrm{Se}_{2} \mathrm{O}_{7}$. Die Einwirkung ist demnach analog der der schwefligen Säure auf $\mathrm{PbO}_{2}$, wodurch die Strukturformel $\mathrm{Pb} \int_{0}^{\mathrm{O}}$ eine neue Bestätigung findet. ${ }^{5}$ Wenn dagegen die Umsetzung mit einer ziemlich konzentrierten Lösung von $\mathrm{H}_{2} \mathrm{SeO}_{3}$ bei Gegenwart von Natriumselenit erfolgt, so wird aus $2 \mathrm{Mol}$. Dioxyd nur 1 Atom Sauerstoff frei und es bindet sich mit der selenigen Säure die Atomgruppierung:

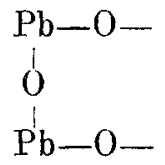

${ }^{1}$ Werner, Neuere Anschauungen auf dem Gebiete der anorgan. Chemie, Braunschweig 1905, S. 176.

2 Hantzsch, Lieb Ann. 291, 317.

"AvgEr, Über einige sauerstoffhaltige Verbindungen des Stickstoffs Ahrens, Sammlung chem. Vortr. (1908), S. 43.

${ }^{4}$ Heinkf, Ber. deutsch. chem. Ges. 31, 1395.

5 Marino, Z. anorg. Chem. 56 (1907), 243. 
Dieses Verhalten lälst an die Möglichkeit der Existenz von Doppelmolekülen $\mathrm{PbO}_{2}$ denken und man könnte das angeführte Beispiel ohne weiteres als einen experimentellen Beitrag für die Isomerie der Dioxyde ansehen, auf die bereits Luther und SchrLow ${ }^{1}$ in ihter sehr interessanten Arbeit: ,Zur Systematik und Theorie gekoppelter Oxydations-Redultionsvorgänge" hingedeutet haben.

Es erscheint deshalb auch der Nachweis interessant, wie die Stabilitätsgebiete der beiden isomeren Formen durch die Konzentration und das Reduktionsvermögen der Säure begrenzt werden, so als ob in konzentrierter Lösung nur Doppelmoleküle $\mathrm{Pb}_{2} \mathrm{O}_{4}$, in verdünnterer dagegen einfache Moleküle $\mathrm{PbO}_{2}$ in Reaktion treten könnten. ${ }^{2}$ - Ich bin mit weiteren Versuchen beschäftigt, die diese Verhältnisse noch anschaulicher beleuchten und das in dieser Mitteilung behandelte Gebiet auf neue Fälle ausdehnen.

1 Ijuther und Schuow, Zeitschr. phys. Chem. 46 (1903), 810.

2 Bei der Unlöslichkeit aller derartigen Verbindungen in den gewöhnlichen Lösungsmitteln, habe ich ihre Molekulargröfse noch nicht bestimmen können, wodurch jedenfalls die beste Aufklärung über die hier behandelten Reaktionen geschaffen werden könnte.

Firenze, Istituto di Chimica Farmaceutica del R. Istituto di Studi Superiori, Juni 1908.

Bei der Redaktion eingegangen am 30. Juni 1908. 